\title{
6 It takes two to tango!: The relevance and dilemma of involving men in the realization of sexual and reproductive health and rights in Africa
}

\author{
Sibusiso Mkwananzi
}

\section{Introduction}

Over the years, efforts have been made to ensure the inclusion of men in policies and programmes towards the realization of sexual and reproductive health and rights in Africa. While the road has been strewn with thorns, modest progress has been made. Experience has shown that the enjoyment by women and girls of their sexual and reproductive health and rights as enshrined in international, regional and national documents may not be possible unless men are viewed as partners in this effort. Often men are regarded as the culprits with regard to the violations of the sexual and reproductive health and rights of women and girls in the region. It is often argued that due to power imbalance and the patriarchal nature of most African societies, men have remained the privileged sex that continue to benefit from this lopsided structure. However, men can equally become important allies in transforming behaviours and advocating for change in societal practices that can advance the sexual and reproductive health and rights of women.

Against this background, this chapter examines the factors that continue to predispose women and girls to sexual and reproductive ill-health in Africa. Using South Africa as a case study, it discusses some of the benefits of involving men in policies and programmes towards the realization of the SRHR of women. In addition, the chapter identifies some of the challenges that may arise in attempting to involve men in programmes to realize SRHR of women. The chapter makes some useful suggestions on the way forward.

\section{Normative framework on sexual and reproductive health and rights}

Sexual and reproductive health and rights are not new sets of rights; rather they are found in existing international and regional human rights instruments.

DOI: 10.4324/9781003175049-6 
For instance, Article 12 of the International Covenant on Economic, Social and Cultural Rights (CESCR) states that all individuals have 'the right to the enjoyment of the highest attainable standard of physical and mental health'. Sexual and reproductive health and rights refer to optimal wellbeing and independence of your body's reproductive system as well as timing, ability, conditions and decision to reproduce. ${ }^{1}$ Specifically, the Committee on Economic, Social and Cultural Rights (CESCR) has developed General Comment 22 on sexual and reproductive health and rights (SRHR) as part of Article 12 and recognizing these as fundamental human rights. Although these rights should have been enjoyed by women as well as men, historically this was not the case, as sexual and reproductive health focused on population control, and thus was linked largely to the policing of women's bodies. ${ }^{2}$

In response, several international norms and standards were developed to ensure women had rights to equality, bodily autonomy, decision making regarding SRHR matters and safety with respect to gender-based violence. ${ }^{3}$ These progressive international norms included Articles 16 and 10 of the Convention on the Elimination of All forms of Discrimination against Women (CEDAW) that ensures women have equal rights to deciding 'freely and responsibly on the number and spacing of their children and to have access to the information, education and means to enable them to exercise these rights' and the right to education should encompass 'access to specific educational information to help to ensure the health and well-being of families, including information and advice on family planning', respectively. Additionally, general recommendation 24 of CEDAW states that nations emphasize the 'prevention of unwanted pregnancy through family planning and sex education', general comment 14 of the CESCR highlights the inclusion of safe pregnancy and childbirth in ensuring a right to health and the Beijing Platform for Action of 1995 concluded that 'the human rights of women include their right to have control over and decide freely and responsibly on matters related to their sexuality, including sexual and reproductive health, free of coercion, discrimination and violence'. ${ }^{4}$ To this end, a stark priority was placed on the specific rights pertaining to women as a marginalized group in most societies, with women disproportionately becoming the focus as well as bearing the responsibility of SRHR in homes and society. ${ }^{5}$ As a result, men seem to have been unintentionally and systematically excluded from this process.

The 1994 Cairo ICPD Conference and Sustainable Development Goal 15 highlight the importance of including men in improving sexual and reproductive health and right (SRHR) globally and this has led to this need being widely accepted by advocates, educators and policy makers. ${ }^{6,7}$ Interventions involving men and boys as crucial partners in improving SRHR across society effectively have accordingly increased globally over the past decade. ${ }^{8}$ However, rates of male partner involvement as well as health-seeking behaviour of men and boys remain low worldwide. ${ }^{9}$ For example, in Southern Africa few men accompany their partners to access sexual and reproductive health services, and HIV testing rates in this group is lower than $50 \% .{ }^{10}$ South African rates of male 
partner involvement and health-seeking behaviour among men and boys remain some of the lowest in the country. ${ }^{11}$ Snow et al $(2010)^{12}$ showed that in rural South African settings, women went for HIV testing at three times higher levels compared to their male counterparts. Additionally, the study by Mohlala et al. $(2011)^{13}$ demonstrated that these challenges occurred regardless of the ruralurban divide, as even in South Africa's urban Cape Town, none of the participant men accompanied their partners to clinics for antenatal services before the intervention. However, this rose to one in three men after the intervention implementation.

South Africa has an estimated population of 57.7 million people, with $49 \%$ of this composing of males. ${ }^{14}$ Although the country fares better developmentally than its counterparts within the Southern African region, some social and reproductive health challenges still remain nationally. ${ }^{15}$ The unemployment rate has been found to range from $20 \%$ to $30 \%$ depending on geographic location. ${ }^{16,17}$ Additionally, although the country's fertility levels have steadily decreased to a total fertility rate (TFR) of 2.4 children per woman currently, this varies greatly by race, and adolescent fertility remains rather high at 50 births per 1000 of 15-19-year-old women. ${ }^{18,19,20,21}$

South Africa has the highest levels of HIV globally with 13 in every 100 individuals (7.52 million) living with HIV. ${ }^{22}$ Correspondingly, there are 1.4 million AIDS orphans nationally, $7 \%$ of who live in child-headed households, and only one in three children live in households with an employed adult. ${ }^{23}$ Many children in South Africa live without their biological father - a direct continued effect of apartheid. Levels of absent yet living fathers rose to $48 \%$ in $2009 .{ }^{24}$ Research has shown that South Africa has one of the highest levels of gender-based violence in the world. ${ }^{25}$ Jewkes et $\mathrm{a}^{26}$ found that one in five young women had encountered more than one incident of physical or sexual intimate partner violence.

In 1994 the reproductive health task force first discovered in their assessment of national sexual and reproductive health (SRH) services that such services do not include men. ${ }^{27}$ Subsequently, the South African government and nongovernmental organizations have created numerous policies and programmes to ensure the inclusion of men and boys in SRH services. For instance with specific reference to HIV services the South African National Strategic Plan on HIV, STIs and TB (NSP), National Action Framework (NAF) for 'No Child Born with HIV by 2015 \& Improving the Health and Wellbeing of Mothers, Partners and Babies in South Africa' and 2010 South African national PMTCT guidelines all directly or indirectly refer to the inclusion of women's partners. Nevertheless, much still needs to be done nationally, as South African studies have shown that women are still more likely than men to attend clinics and initiate treatment after being diagnosed with HIV. ${ }^{28,29}$ Therefore, it is important to explore the challenges that surround this important move.

This chapter aimed to describe:

1. What previous studies have found are the benefits and difficulties of involving men in SRHR work 
2. The challenges that underlie men being 'left behind' in conversations and advancement of SRHR

3. Ways in which men could be involved in SRHR work

\section{Effects of involving men in SRHR}

The first question becomes why does the inclusion of men in sexual and reproductive health remain important? For the purpose of this chapter, involving men in SRHR is limited to the individual level. Also, the chapter only provides examples and implementation of involving men in SRH work that encompasses prevention of sexually transmitted infections (STIs) including HIV, prevention of maternal mortality, anti-gender based violence (GBV) initiatives as well as contraceptive use. Previous studies from developing countries have shown large bodies of empirical evidence to suggest that involving men (i.e. individual partners) in SRHR work has advantages as well as disadvantages for the men in question, their partners, the couple as a unit and even subsequent offspring as will be discussed further below.

\subsection{The advantages of involving men}

Benefits for men incorporated in South African and Ugandan programmes aimed at engaging them on sexual and reproductive health matters include less risky sexual decisions that encompass increased condom use, delayed sexual debut as well as a decreasing likelihood of multiple concurrent sexual partners. ${ }^{30,31,32}$ Additionally, Reddy et al $(2014)^{33}$ established that involving men in reproductive health decisions led to the prevention of STI \& HIV/AIDS and improved reproductive health of men in such programmes in South Africa.

Nevertheless, the effects of including men go beyond the individual male included, having advantages for their partner as well. Such partner gains encompass increased support of their partner's needs and higher levels of escorting of partners to clinic to access SRH services including termination of pregnancy management as evidenced in Uganda and Asia. ${ }^{34,35}$ Overall increased levels of maternal health have also been shown to occur through men's participation in Malawi resulting in increased uptake of antenatal and postnatal care, birth preparedness, lower maternal workload while expecting and decreased maternal stress. ${ }^{36}$ Another dimension to partner benefits are the added advantages that come with changed attitudes amongst men that promote gender equality and decrease tolerance to gender based violence that can lead to increased enthusiasm and conscientiousness towards conducting domestic chores as shown in the East African study. ${ }^{37}$ Similarly, in Iran most studies testing the influence of men's awareness and involvements have shown a positive effect on women's SRH. ${ }^{38}$ Another evaluation of initiatives that included men in Sierra Leone, Cote d'Ivoire, Liberia and the Democratic Republic of Congo (DRC) found reduced levels of gender based violence (both sexual and physical forms) as well as improved views on men's use of violence. ${ }^{39}$ Although such an advantage is immediately experienced by one's partner, greater society could also 
subsequently benefit from these changed attitudes. Therefore, it becomes particularly important in the context of South Africa where $20 \%-40 \%$ of women (depending on locality) have experienced some form of violence in their lifetime. ${ }^{40}$

Gains for the couple have also been suggested as Zamawe et al. (2015) ${ }^{41}$ showed that engagement of men was associated with increased use of family planning and contraceptives in Malawi. Similarly, a higher likelihood of open discussion with partners about reproductive planning and decision making were said to occur in Uganda after engaging men on SRHR matters. ${ }^{42}$

Benefits for the children born include higher levels of child health with reduced likelihood of preterm births, low birth weight, foetal growth restriction and infant mortality. ${ }^{43}$ This may be linked to the ability of male engagement to increase access and uptake of new-born and child health services as shown in rural India. ${ }^{44}$ Additionally, Reddy et al $(2016)^{45}$ in Asia showed that men who were involved in reproductive health decisions have a stronger bond with their offspring. This of course has been determined to have future benefits for the child regarding self-esteem, confidence and the ability to achieve better life outcomes in general. ${ }^{46,47}$ Thus, what can be deduced from the discussion here is that involving men in SRH issues can boost family bond and congeniality thereby advancing the realization of women's rights as well as enhancing their reproductive autonomy and bodily integrity.

Despite the benefits of involving men in SRHR matters, it is important to ensure that this does not undermine women's rights to choice and autonomy. In essence, while it is desirable for men to be involved in decision-making relating to SRH, women should still be accorded the autonomy to decide whether to involve their partners on this issue. Thus, questions may be asked: what should happen when women do not want their male partners involved? Where should the line be drawn to ensure that the rights and responsibilities of both parties engaged in sexual relations are honoured? Some of these issues are discussed in the next section.

\subsection{The difficulties of involving Men}

Despite the advantages of involving men in SRH matters as discussed above, there are some challenges associated with this. As scholars and programme implementers, it is evident that involving men in SRHR is important, yet this continues to be conducted in exceptional cases. Beyond the dilemma occurring among scholars and programmers, previous work in different settings has also highlighted some difficulties to involving men in SRHR. These include:

First and foremost traditional gender norms exist at the societal level. ${ }^{48}$ These reveal themselves through men not being interested in their own SRH or that of their partners as the subject is generally regarded as a 'woman's issue' ${ }^{49}$ Research in South Africa has reported that even when men want to be involved they may be scrutinized and giggled at by other female clients, reprimanded and chased out by health workers on site, as well as made to 
feel uncomfortable by male-unfriendly clinic environments. ${ }^{50}$ The restrained contraceptive, health service delivery and reproductive health awareness campaign options specifically designed for men in Uganda further exacerbate these problematic societal norms. ${ }^{51}$ Therefore, addressing this challenge will require community-wide education as well as more resources for the shifting of social and institutional gender norms. ${ }^{52}$ The examples from above highlight the need for institutional buy-in for the accommodation of men in SRH issues in order to decrease stigma. Additionally, clinic and hospital staff members exhibiting discriminatory practices and prejudices against male partners may invariably be infringing their rights to dignity, equality and freedom.

Over and above societal norms are masculine-related convictions, beliefs and attitudes that prevent HIV testing, treatment and care among men, hinder the involvement of men in SRH services as well as promote negative views about family planning. ${ }^{53}$ Many of these views stem from cultural and traditional patriarchal paradigms that disallow men to show signs of weakness, uncertainty, loss of confidence and vulnerability. ${ }^{54}$ This can even lead to an inability among some men to share their emotions in all-male environments making the creation of safe male dialogue platforms difficult. ${ }^{55,56}$ To address these gender biases, it is necessary to reform school curriculum on SRHR so that rights and responsibilities are assigned to both girls and boys at an early age.

Over and above this, the reforming of societal and individual masculinerelated norms will require the focus of women in national policies and health systems across Africa to change. Mullick et al. (2005) ${ }^{57}$ suggest that successful male involvement will occur as health infrastructure addresses questions of accommodating employed men, the unmet need of male contraceptive methods, men-friendly SRH services as well as designing comprehensive health packages that couples and families can attend simultaneously. Lack of access to SRHR services for men results in a systematic violation of the right to SRHR that is in breach of a state obligation under international law. Therefore, it is important that an enabling policy environment be created to encourage and support the presence and participation of men in SRH. This would also need to include a possible change in the language of SRH messaging in order to make it less centred on women. All individuals are accorded the right to information, including information for correct decision making as regards SRH.

\section{Challenges that underlie not involving men in SRHR work}

The second question that arises is that in spite of the above advantages, why does a reluctance of including men in SRHR work still exist? The general notion seems to be that SRHR scholars and programme implementers want to include men, but do not want to end up regretting this stance. Numerous elements of this notion could answer the question stated for this section, and the next section unpacks a few of these as well as attempts to give solutions as a means of progressing forward. 
The concern that comes to mind initially is the fear that involving men may change largely female-led and pro-partner SRHR current work into male-led, pro-man SRHR campaigns. This would lead to SRHR work losing its drive to promote women's health and rights and the objectives for having started such programmes that involve men will no longer be achieved. For example a ten-year study evaluating programmes that engaged men in groups in Sierra Leone, Cote d'Ivoire, DRC and Tanzania, showed a consistent straying from violence against women and girls to discussions on the struggles that men face. Facilitators also found it difficult to sway the focus back to its primary aim. ${ }^{58}$

Slightly related to the point above is the notion that if SRHR work is to include male leaders and train them up to be advocates in this field, what if they are then found to be in violation of the very principles that they were advocating for? This concern is rather valid as seen in the recent case of a prominent advocate of women's rights who was accused of sexual harassment, sexism, transphobia and homophobia. ${ }^{59}$ Similarly, this challenge occurred when transformative gender programmes that aimed to involve men in West, East and Central Africa led to male domination of mixed-group discussions, silencing of women as well as facilitators struggling to address verbal and mental resistance from male participants. In response to these first fears, possibly the onus is on us as female advocates of SRHR to ensure not only that we lead programmes that involve men, but that we monitor and stay attached to such programmes for as long as possible so as they adhere to the original ethos of the programme's spirit of being pro-partner if male advocates have been capacitated to lead such sessions. Additionally, we can never be completely sure of people's character, be they male or female, and hence this is a risk we are going to have to take as we embark upon this journey. Knowing the risk in advance though may assist us to ensure that we catch any worrying signs earlier on, and it is comforting to know that such cases are rather the exception than the rule in the field of SRHR advocacy work.

The hashtag movements of \#metoo and \#churchtoo, where over the past year there has been a rising level of women, men and boys coming forward and speaking against widespread outcry against sexual harassment and violence in the film industry as well as the church, has revealed how widespread the abuse of power is. The alleged perpetrators in the above scenarios occupying these positions of power have largely been men. However, maybe such programmes could be opportunities to change the mind-set of powerful men to see the importance of SRHR personally for themselves and to become actively engaged in the well-being of their partners and their reproductive health. This could possibly lead to a drop in gender-based violence and increase gender understanding. Additionally, as structural drivers have been repeatedly identified as the factors that hinder gender equality and SRHR health, men in positions of power could use the resources and influence available to them to ensure effective redress. An example of this having worked well is the training and education of traditional chiefs and municipal leaders in Zambia in child marriage, that has led to the criminalization of it and more cases of child 
marriage being addressed at local traditional courts with subsequent reporting to and arrest by police. ${ }^{60,61}$ Hence, it becomes no surprise that child marriage is on the decline even in rural areas in Zambia. ${ }^{62,63}$

Also, some male scholars and programme implementers may ask: to what extent would men be involved, as many feminist researchers have shown tendencies of no longer wanting to engage with men in an attempt to deconstruct patriarchal systems and ideologies? The approach of including men is in fact a feminist stance due to it advocating for gender equality education and awareness among men. Additionally, it is important to note that feminism has never been against men, but rather has advocated against the unfair treatment of women regardless of race, culture, age, etc. ${ }^{64}$ Additionally, the approach of educating on gender equality is not about emasculating men but rather discouraging hegemonic and aggressive masculinities to decrease violence perpetration as well as risky sexual behaviours. However, caution should be taken in order not to overemphasize this element in the pursuit of progressive and transformative masculinities. How do men and boys enter this SRHR debate? Is it our desire to label them as the problems or as part of the solution? As scholars and programme implementers, we will have to decide carefully how to frame men in the SRHR conversation in order to keep them as willing allies. It is imperative that we do not alienate men, particularly in African contexts through problematizing manhood and all aspects of masculinity.

The same goes for problematizing culture too harshly. SIHA $(2016)^{65}$ speak of 'In many African societies, challenging masculinities and negative male norms can meet with resistance and taboo'. The secret statuses accorded to these rituals also 'mystify' masculinity and may contribute to the normalization of men's violent behaviour towards women. Furthermore, there are incredibly misogynistic ideologies attached to the defence of certain traditional rites (of passage) and rituals practiced by and on men. It is important not to throw out the baby with the bathwater in such cases as are mentioned here. Rituals associated with the rites of passage into adulthood are performed for men and women in African cultures. ${ }^{66,67}$ Additionally, the rituals themselves do not make men violent, but rather it is the teachings and messages that accompany the rituals which may promote misogyny, the performance of hegemonic masculinities and subordination of women. Therefore, eliminating traditions completely is a rather colonial approach and should not be advocated, rather we need to remember that culture is forever evolving and therefore allows leeway to be adapted to be less harmful when the appropriate steps are taken.

Finally, the question can be asked at what cost do we have to include men and is this the smartest approach to use? It is important to involve men without excluding other activities. In other words, this should be added as one of the strategies that we pursue to ensure SRHR for women without abandoning those already present. Particularly, the idea of replacing women-geared programmes with men-geared programmes is dangerous. Indeed it is the other side of the relationship; however, the approach may assist researchers to reach more relationships as well. be they same-sex or heterosexual relationships. Additionally, 
a feedback mechanism where good and bad components of programmes are reported back to the implementers will be useful to determine where modification of programmes is necessary. Consequently, despite the above numerous challenges being valid and making it understandable why female scholars and programme implementers may deliberately choose to exclude men in their work, a strong case can still be made for including men in SRHR.

\section{The way forward}

\subsection{Ways in which men can be involved in SRHR work}

The last question then becomes in what ways could men be involved in SRHR work? There are three areas to consider as proposed by the male involvement model of Greene et al. (2006). ${ }^{68}$

Men as clients: this component argues the need to increase the number of men accessing SRH services. Ensuring the presence of sexual and reproductive health infrastructure that targets men, including policies, services and opening hours is vital. Such services should incorporate: SRH knowledge, family planning, HIV and other STI testing, VMMC, urologist referral for complicated cases as well as prostate check-ups.

Men as partners: this component argues the need to educate and increase awareness among men to ensure optimal SRHR for their partners. Although women remain the custodians of decision-making regarding their bodies since they enjoy autonomy, men can be empowered to take up supportive role. This involves advocacy and education via workshops, posters and community dialogues to change gender inequitable attitudes and perceptions among men; this would eliminate the tolerance of violence against women and teach men the SRH rights that women have (external and internal to relationship). This aspect aims to increase: communication about sexual decision-making and choosing family planning options, supporting women's initiation of contraception, escorting their partners to access SRHR services and antenatal care as well as postnatal care. In as much as men are partners in this process, the ultimate and final decision lies with the woman in accordance with the rights of women to equality, dignity, and respect for private life, without discrimination by the OHCHR. ${ }^{69}$

Men as advocates of change: this element argues the need to educate and increase awareness among men that supports men to mobilize other men's involvement in sexual and reproductive health.

\section{Conclusion}

The opportunity to include men in sexual and reproductive health and rights advancement of women remains full of positive rewards. This article has attempted to show the benefits and challenges, reasons for reluctance as well as possible ways that the move could be implemented on the ground. Risks may 
still occur as shown in the paper. However, the benefits seem to far outweigh these and hence programmes should be modified for contextual factors as they are implemented in various African settings. Zusak $(2018)^{70}$ states that 'Like everything else worth fighting for in our lives, the idea of raising good men feels to me like something that never ends. It will to and fro between triumphs and failures...' This is the attitude that we need to have as we continue to include men in SRH services. It is important to acknowledge and accept that things will not happen rapidly, as attitudes and behaviour change are slow processes regardless of gender. ${ }^{71}$ Nevertheless as the title of this chapter rightly states, it does take two to tango. Heterosexual relationships do not involve women alone. Consequently, it is when we include men consistently in SRH service conversations and work that there will be strides in changing mind-sets and behaviour to achieve optimal sexual and reproductive health and rights for women and men in sub-Saharan Africa.

\section{Notes}

1 United Nations Population Fund, Sexual and reproductive health UNFPA https://www .unfpa.org/sexual-reproductive-health (accessed 20 April 2020).

2 MenEngage Alliance 'Accountability training toolkit' (2018) http://menengage.org/ resources/menengage-accountability-training-toolkit/ (accessed 20 April 2020).

3 Office of the High Commissioner for Human Rights (OHCHR), Sexual and reproductive health and rights UN Human Rights https://www.ohchr.org/en/issues/women/ wrgs/pages/healthrights.aspx (accessed 20 April 2020).

4 As above.

5 MenEngage (n 2).

6 R Jewkes et al 'From work with men and boys to changes of social norms and reduction of inequities in gender relations: A conceptual shift in prevention of violence against women and girls' (2015) 385 The Lancet 1580.

7 E Stern et al 'Lessons learned from engaging men in sexual and reproductive health as clients, partners and advocates of change in the Hoima district of Uganda' (2015) 17 Culture, Health \& Sexuality 190.

8 Jewkes et al. (n 6).

9 P Fleming et al 'What role can gender-transformative programming for men play in increasing men's HIV testing and engagement in HIV care and treatment in South Africa?' (2016) 18 Culture, Health \& Sexuality 1251.

10 K Koo, J Makin \& B Forsyth 'Barriers to male-partner participation in programs to prevent mother-to-child HIV transmission in South Africa' (2013) 25 AIDS Education and Prevention 14.

11 W Van Den Berg et al 'Improving men's participation in preventing mother-to-child transmission of HIV as a maternal, neonatal, and child health priority in South Africa' (2015) 12 PLoS Medicine e1001811.

$12 \mathrm{R}$ Snow et al 'Are men testing? Sex differentials in HIV testing in Mpumalanga province, South Africa' (2010) 22 AIDS Care 1060.

13 B Mohlala et al 'The forgotten half of the equation: Randomized controlled trial of a male invitation to attend couple voluntary counselling and testing' (2011) 25 AIDS 1535.

14 Statistics South Africa; in bibliography StatsSA, 'Mid-year population estimates' (Statistics South Africa, 2018).

15 Van den Berg et al (n 11). 
16 G Kingdon \& J Knight 'Race and the incidence of unemployment in South Africa' (2004) 8 Review of Development Economics 198.

17 S Klasen et al 'Surviving unemployment without state support: Unemployment and household formation in South Africa' (2009) 18 Journal of African Economies 1.

18 S Panday et al Teenage pregnancy in South Africa: With a specific focus on school-going learners (2009) (Department of Basic Education).

19 L Rossouw et al 'The fertility transition in South Africa: A retrospective panel data analysis' 29 (2012) Development Southern Africa 738.

20 United Nations World Population Prospects: The 2012 Revision (2012).

21 Statistics South Africa (n 14).

22 Joint United Nations Programme on HIV/Aids 'UNAIDS report on the global AIDS epidemic 2013' (Geneva: UNAIDS, 2013 (2014)).

23 L Holborn \& G Eddy 'First steps to healing the South African family' (South African Institute of Race Relations, 2011).

24 As above.

25 E Durojaye 'Access to contraception for adolescents in Africa: A human rights challenge' (2011) 44 Comparative and International Law Journal of Southern Africa 1.

$26 \mathrm{R}$ Jewkes et al 'Intimate partner violence, relationship power inequity, and incidence of HIV infection in young women in South Africa: A cohort study' (2010) 376 The Lancet 41.

27 Reproductive Health Task Force, 'Assessment of reproductive health services in South Africa, focusing on family planning' (South African Ministry of Health and WHO-HRP, 1994).

28 D Govindasamy et al 'Linkage to HIV care from a mobile testing unit in South Africa by different CD4 count strata' (2011) 58 Journal of Acquired Immune Deficiency Syndromes (1999) 344.

29 K Kranzer et al 'Linkage to HIV care and antiretroviral therapy in Cape Town, South Africa' (2010) 5 PLoS One e13801.

30 F Cornish \& C Campbell 'The Social conditions for successful peer education: A comparison of two HIV prevention programs run by sex workers in India and South Africa' (2009) 44 American Journal of Community Psychology 123.

31 A Foss et al 'A systematic review of published evidence on intervention impact on condom use in sub-Saharan Africa and Asia' (2007) Sexually Transmitted Infections 1.

32 Stern (n 7).

33 D Reddy et al 'Participation of men in reproductive health matters: A comparative study in rural and urban areas' (2014) 1 Asia Pacific Journal of Research 7.

34 As above.

35 Stern (n 7).

36 C Zamawe et al 'The effect of mass media campaign on men's participation in maternal health: A cross-sectional study in Malawi' (2015) 12 Reproductive Health 31.

37 Stern (n 7).

$38 \mathrm{~S}$ Khani 'A review on various aspects of male involvement in women's sexual and reproductive health’ 27 (2017) Journal of Mazandaran University of Medical Sciences 99.

39 International Rescue Committee 'Preventing violence against women and children: Engaging men through accountable practice' (2013) https://www.fsnnetwork.org/sites /default/files/IRC-EMAP-Introductory-Guide-High-Res\%20\%281\%29.pdf (accessed 25 May 2020).

40 M Merten 'Statistics SA: One in five SA women experience physical violence, young women hard-hit by HIV/Aids' (2017) Daily Marverick https://www.dailymaverick.co.za /article/2017-05-15-statistics-sa-one-in-five-sa-women-experience-physical-violenceyoung-women-hard-hit-by-hivaids/ (accessed 15 August 2018).

41 Zamawe (n 36).

42 Stern (n 7).

43 Zamawe (n 36). 
$44 \mathrm{~J}$ Fotso et al 'Male engagement as a strategy to improve utilization and community-based delivery of maternal, newborn and child health services: Evidence from an intervention in Odisha, India' 15 (2015) BMC Health Services Research 1.

45 Reddy (n 33).

46 D Franklin 'Race, class, and adolescent pregnancy: An ecological analysis' (1988) 58 American Journal of Orthopsychiatry 339.

$47 \mathrm{~J}$ Luo et al 'The influence of the absence of fathers and the timing of separation on anxiety and self-esteem of adolescents: A cross-sectional survey' (2012) 38 Childcare, Health and Development 723.

48 S Mullick et al 'Involving men in maternity care: Health service delivery issues' 6 (2005) Agenda Special Focus 124.

49 A Kabagenyi et al 'Barriers to male involvement in contraceptive uptake and reproductive health services: A qualitative study of men and women's perceptions in two rural districts in Uganda' (2014) 11 Reproductive Health 21.

50 Mullick et al (n 48).

51 Kabagenyi et al (n 49).

52 As above.

53 Fleming (n 9).

54 As above.

55 As above.

56 O Stern et al "Working with men and boys: Emerging strategies from across Africa to address gender- based violence and HIV/AIDS' (2009) http://paulkivel.com/wpcontent/uploads/2015/07/menengage_casestudies.pdf (accessed 21 May 2020).

57 Mullick (n 48).

58 International Rescue Committee.

59 R Ratcliffe 'Eminent sociologist Michael Kimmel steps down from board of gender equality campaign group' The Guardian (2018) https://www.theguardian.com/global -development/2018/aug/15/us-womens-rights-campaigner-accused-of-sexual-harassment (accessed 15 August 2018).

60 N Coulson et al 'Midterm review-REPSSI strategy 2016-2020' (2018) 61.

61 V Wang 'Ending child marriages - new laws bring progress but hurdles remain' (2016) http://hdl.handle.net/11250/2475409 (accessed 15 August 2018).

62 Coulson et al (n 60).

63 The DHS Program, Zambia Ministry of Health and Icf International 'Zambia Demographic and health survey 2013-2014' (Central Statistical Office, 2014).

64 M Beksinska et al 'Sexual and reproductive health and rights: Reflections on the millennium development goals' (2010) South African Health Review 33.

65 SIHA 'Negotiating patriarchy: Men as allies against violence against women' (2016) http://sihanet.org/faq/negotiating-patriarchy-men-as-allies-against-violence-against -women/ (accessed 16 August 2018).

66 G Herdt Rituals of manhood (2017).

67 E Salo Respectable mothers, tough men and good daughters: Producing persons in Manenberg township South Africa (2018).

68 M Greene et al Involving men in reproductive health: Contributions to development (2006).

69 Office of the High Commissioner for Human Rights, 'Women's autonomy, equality and reproductive health in international human rights: Between recognition, backlash and regressive trends (2017) https://www.ohchr.org/Documents/Issues/Women/WG/ WomensAutonomyEqualityReproductiveHealth.pdf (accessed 12 August 2018).

$70 \mathrm{M}$ Zusak $A$ love that is big and big hearted (2018) 57.

71 B Burnes 'Kurt Lewin and the planned approach to change: A re-appraisal' (2004) 41 Journal of Management Studies 977. 


\section{References}

Beksinska, M, Ramikisson, A, Searle, C \& Burns, C 'Sexual and reproductive health and rights: Reflections on the millennium development goals' (2010) 20 South African Health Review 33.

Burnes, B 'Kurt Lewin and the planned approach to change: A re-appraisal' (2004) 41 Journal of Management Studies 977.

Canadian Civil Liberties Association 'Summary: International covenant on civil and political rights Toronto: Canadian Civil Liberties Association' https://ccla.org/summary -international-covenant-on-civil-and-political-rights-iccpr/ (accessed 20 April 2020).

Cornish, F \& Campbell, C 'The social conditions for successful peer education: A comparison of two HIV prevention programs run by sex workers in India and South Africa' (2009) 44 American Journal of Community Psychology 123.

Coulson, N Midterm review-REPSSI strategy 2016-2020 (Department of Health 2018).

Durojaye, E 'Access to contraception for adolescents in Africa: A human rights challenge' (2011) 44 Comparative and International Law Journal of Southern Africa 1.

Fleming, P, Colvin, C, Peacock, D \& Dworkin, S 'What role can gender-transformative programming for men play in increasing men's HIV testing and engagement in HIV care and treatment in South Africa?' (2016) 18 Culture, Health \& Sexuality 1251.

Franklin, D 'Race, class, and adolescent pregnancy: An ecological analysis' (1988) 58 American Journal of Orthopsychiatry 339.

Foss, A 'A systematic review of published evidence on intervention impact on condom use in sub-Saharan Africa and Asia' (2007) 87 (3) Sexually Transmitted Infections 510.

Fotso, J, Higgins-Steele, A \& Mohanty, S 'Male engagement as a strategy to improve utilization and community-based delivery of maternal, newborn and child health services: Evidence from an intervention in Odisha, India' (2015) 15 BMC Health Services Research 1.

Govindasamy, D, Schaik, N, Kranzer, K, Wood, R, Matthews, C \& Bekker, L 'Linkage to HIV care from a mobile testing unit in South Africa by different CD4 count strata' (2011) 58 Journal of Acquired Immune Deficiency Syndromes 344.

Greene, M, Mehta, M, Pulerwitz, J, Wulf, D, Bankole, A \& Singh, S Involving men in reproductive health: Contributions to development (UN Millenium Project 2006).

Herdt, G Rituals of manhood (Routledge 2017).

Holborn, L \& Eddy, G First steps to healing the South African family (South African Institute of Race Relations 2011).

Jewkes, R, Dunkie, K, Nduna, M \& Shai, N 'Intimate partner violence, relationship power inequity, and incidence of HIV infection in young women in South Africa: A Cohort study' (2010) 376 The Lancet 41.

Jewkes, R, Flood, M \& Lang, J 'From work with men and boys to changes of social norms and reduction of inequities in gender relations: A conceptual shift in prevention of violence against women and girls' (2015) 385 The Lancet 1580.

Kabagenyi, A 'Barriers to male involvement in contraceptive uptake and reproductive health services: A qualitative study of men and women's perceptions in two rural districts in Uganda' (2014) 11 Reproductive Health 21.

International Rescue Committee 'Preventing violence against women and children: Engaging men through accountable practice' (2013) https://www.fsnnetwork.org/sites /default/files/IRC-EMAP-Introductory-Guide-High-Res\%20\%281\%29.pdf (accessed 10 April 2020).

Khani, S 'A review on various aspects of male involvement in women's sexual and reproductive health' (2017) 27 Journal of Mazandaran University of Medical Sciences 99. 
Kingdon, G \& Knight, J 'Race and the incidence of unemployment in South Africa' (2004) 8 Review of Development Economics 198.

Klasen, S \& Woolard, I 'Surviving unemployment without state support: Unemployment and household formation in South Africa' (2009) 18 Journal of African Economies 1.

Koo, K, Makin, J \& Forsyth, B 'Barriers to male-partner participation in programs to prevent mother-to-child HIV transmission in South Africa' (2013) 25 AIDS Education and Prevention 14.

Kranzer, K 'Linkage to HIV care and antiretroviral therapy in Cape Town, South Africa' (2010) 5 Plos One e13801.

Luo, J, Wang, L \& Gao, W, 'The influence of the absence of fathers and the timing of separation on anxiety and self-esteem of adolescents: A cross-sectional survey' (2012) 38 Child: Care, Health and Development 723.

Mohlala, B, Boily, M \& Gregson, S 'The forgotten half of the equation: Randomized controlled trial of a male invitation to attend couple voluntary counselling and testing' (2011) 25 AIDS 1535.

MenEngage Alliance 'Accountability training toolkit' (2018) http://menengage.org/ resources/menengage-accountability-training-toolkit/ (accessed 15 April 2020).

Merten, M 'Statistics SA: One in five SA women experience physical violence, young women hard-hit by HIV/Aids' (2017) Daily Marverick https://www.dailymaverick.co.za /article/2017-05-15-statistics-sa-one-in-five-sa-women-experience-physical-violence -young-women-hard-hit-by-hivaids/ (accessed 17 April 2020).

Mullick, S, Kunene, B \& Wanjiru, M 'Involving men in maternity care: Health service delivery issues' (2005) 6 Agenda Special Focus 124.

Panday, S Teenage pregnancy in South Africa: With a specific focus on school-going learners (Department of Basic Education 2009).

Ratcliffe, R 'Eminent sociologist Michael Kimmel steps down from board of gender equality campaign group' The Guardian (London, United Kingdom), 15 August 2018 https://www.theguardian.com/global-development/2018/aug/15/us-womens -rights-campaigner-accused-of-sexual-harassment (accessed 15 April 2020).

Reddy, D, Chandrasekarayya, T \& Murthy, P 'Participation of men in reproductive health matters: A comparative study in rural and urban areas' (2014) 1 Asia Pacific Journal of Research 7.

Reproductive Health Task Force Assessment of reproductive health services in South Africa, focusing on family planning (South African Ministry of Health and WHO-HRP 1994).

Rossouw, L \& Burger, R 'The fertility transition in South Africa: A retrospective panel data analysis' (2012) 29 Development Southern Africa 738.

Salo, E Respectable mothers, tough men and good daughters: Producing persons in manenberg township South Africa (Langaa Research and Publishing CIG 2018) .

Stern, E, Pascoe, L, Shand, T, \& Richmond, S 'Lessons learned from engaging men in sexual and reproductive health as clients, partners and advocates of change in the Hoima district of Uganda' (2015) 17(Supplement 2) Culture, Health \& Sexuality 190.

Stern, O, Peacock, D \& Alexander, H Working with men and boys: Emerging strategies from across Africa to address gender-based violence and HIV/AIDS (Sonke Gender Justice Network 2009).

Snow, R, Madalane, M \& Poulsen, M 'Are men testing? Sex differentials in HIV testing in Mpumalanga province, South Africa' (2010) 22 AIDS Care 1060.

SIHA Negotiating patriarchy: Men as allies against violence against women (Kampala: SIHA 2016) 38. http://sihanet.org/faq/negotiating-patriarchy-men-as-allies-against-violence-against -women/ (accessed 15 April 2020).

Statssa Mid-year population estimates (Pretoria: Statistics South Africa 2018). 


\section{Sibusiso Mkwananzi}

The Dhs Program, Zambia Ministry of Health and Icf International Zambia demographic and health survey 2013-2014 (Lusaka: Central Statistical Office 2014).

United Nations World population prospects: The 2012 revision (United Nations 2012).

United Nations Office of the High Commissioner for Human Rights Sexual and reproductive health and rights (New York: UN Human Rights) https://www.ohchr.org/en/issues/ women/wrgs/pages/healthrights.aspx (accessed 20 April 2020).

United Nations Office of the High Commissioner for Human Rights Women's autonomy, equality and reproductive health in international human rights: Between recognition, backlash and regressive trends (New York 2017) https://www.ohchr.org/Documents/Issues/Women /WG/WomensAutonomyEqualityReproductiveHealth.pdf (accessed 20 April 2020).

United Nations Population Fund Sexual and reproductive health (New York: UNFPA) https:// www.unfpa.org/sexual-reproductive-health (accessed 20 April 2020).

Van Den Berg, W 'Improving men's participation in preventing mother-to-child transmission of HIV as a maternal, neonatal, and child health priority in South Africa' (2015) 12 PLoS Medicine e1001811.

Wang, V Ending child marriages — new laws bring progress but hurdles remain (Germany: Chr. Michelsen Institute 2016) http://hdl.handle.net/11250/2475409 ( accessed 20 April 2020).

Zamawe, C, Banda, $M \&$ Dube, A 'The effect of mass media campaign on men's participation in maternal health: A cross-sectional Study in Malawi' (2015) 12 Reproductive Health 31.

Zusak, M A love that is big and big hearted (Johannesburg: Sunday Times 2018). 